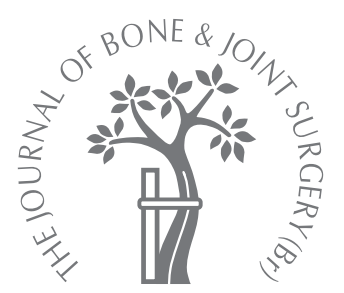

P. K. Jaiswal, J. Jagiello, L. A. David, G. Blunn, R. W. J. Carrington, J. A. Skinner, S. R. Cannon, T. W. R. Briggs

From Royal National Orthopaedic Hospital Trust, Stanmore, England

P. K. Jaiswal, MRCS Specialist Registrar

J. Jagiello, MRCS, Specialist Registrar

L. A. David, FRCS(Tr \& Orth), Specialist Registrar

In. Blunn, PhD, Professor of Biomedical Engineering R. W. J. Carrington, FRCS(Tr \& Orth), Consultant

Orthopaedic Surgeon

J. A. Skinner, FRCS(Tr \&

Orth), Consultant Orthopaedic

Surgeon

S. R. Cannon, FRCS

Consultant Orthopaedic

Surgeon

T. W. R. Briggs, FRCS

Consultant Orthopaedic

Surgeon

Royal National Orthopaedic Hospital Trust, Brockley Hill,

Stanmore, Middlesex HA7 4LP,

UK.

Correspondence should be sent to Mr P. K. Jaiswal at 36 Rothsay Street, London SE1 4UE, UK; e-mail:

pkjaiswal05@googlemail.com

(C)2008 British Editorial Society of Bone and Joint Surgery doi:10.1302/0301-620X.90B1. $19494 \$ 2.00$

$J$ Bone Joint Surg $[\mathrm{Br}]$ 2008;90-B:11-15.

Received 28 March 2007;

Accepted after revision 24

August 2007

\title{
Use of an 'internal proximal femoral replacement' with distal fixation in revision arthroplasty of the hip
}

\begin{abstract}
We have managed 27 patients (16 women and 11 men) with a mean age of 68.4 years (50 to 84), with failed total hip replacement and severe proximal femoral bone loss by revision using a distal fix/proximal wrap prosthesis. The mean follow-up was for $\mathbf{5 5 . 3}$ months (25 to 126). The mean number of previous operations was 2.2 ( 1 to 4 ). The mean Oxford hip score decreased from 46.2 (38 to 60 ) to 28.5 (17 to 42 ) (paired t-test, $p<0.001$ ) and the mean Harris Hip score increased from 30.4 (3 to 57.7) to 71.7 (44 to 99.7) (paired t-test, $p<0.001$ ). There were two dislocations, and in three patients we failed to eradicate previous infection. None required revision of the femoral stem.

This technique allows instant distal fixation while promoting biological integration and restoration of bone stock. In the short term, the functional outcome is encouraging and the complication rates acceptable in this difficult group of patients.
\end{abstract}

Loss of femoral bone in revision arthroplasty of the hip is a challenging reconstructive problem. Surgical options include the use of a fully-cemented long canal-filling femoral stem, uncemented distal fixation with or without locking screws and proximal wrap, a proximal allograft-prosthesis composite, or a customised or modular proximal femoral endoprosthetic replacement, which will usually result in considerable muscle detachment with loss of function and stability. In Paprosky grade IIIB and IV defects, ${ }^{1}$ obtaining fixation and rotational stability with the above constructs can be difficult. ${ }^{2}$ Grade IIIB defects are characterised by a severely-damaged metaphysis, with less than $4 \mathrm{~cm}$ of diaphyseal bone available for distal fixation. ${ }^{3}$ Such a defect is often seen following failure of a cemented femoral component which was inserted with a cement restrictor, or a cementless component associated with substantial distal osteolysis. ${ }^{4}$ Type IV defects in which there is extensive metadiaphyseal damage and a widened femoral canal are rare. The isthmus is non-supportive and uncemented distal fixation cannot be achieved. ${ }^{4}$

We have designed and developed a custommade 'internal proximal femoral replacement' prosthesis that allows cemented distal fixation, retaining the abductors and the remaining proximal femoral bone stock in patients with Paprosky grade IIIB and IV defects. We have extended the indication for this prosthesis to difficult peri-prosthetic fractures (Vancouver type $\mathrm{B} 3^{5}$ ). We describe the operative technique and the early clinical and radiological results.

\section{Patients and Methods}

This prospective study analysed all 27 patients who underwent internal proximal femoral replacement for failed hip replacement between April 1996 and April 2005 in our centre. The indications were a failed hip arthroplasty with a Paprosky grade IIIB/IV defect of the proximal femur, or a difficult periprosthetic fracture with proximal bone loss. The operations were performed by the four senior authors (RWJC, JAS, SRC and TWRB). The prosthesis is manufactured by Stanmore Implants Worldwide (Stanmore Implants Worldwide Ltd, Stanmore, United Kingdom) and is composed of a vanadium, titanium and aluminium alloy. It has a hydroxyapatite (HA)-coated collar of variable length and the proximal part is also HA-coated (Fig. 1). The stem distal to the collar is tapered and cemented. The HA coating conforms to the following criteria: thickness $80 \mathrm{~mm}$ to 120 $\mathrm{mm}$, bond strength 20 Megapascals (MPa) to $40 \mathrm{MPa}$, X-ray diffraction purity $97 \%$, crystallinity $92 \%$ to $96 \%$. An option for a distal extracortical plate is available if there is poor fixation with the stem alone.

There were 11 men and 16 women. Their mean age at operation was 68.4 years $(50$ to 84). A mean of 2.2 ( 1 to 4 ) previous operations 


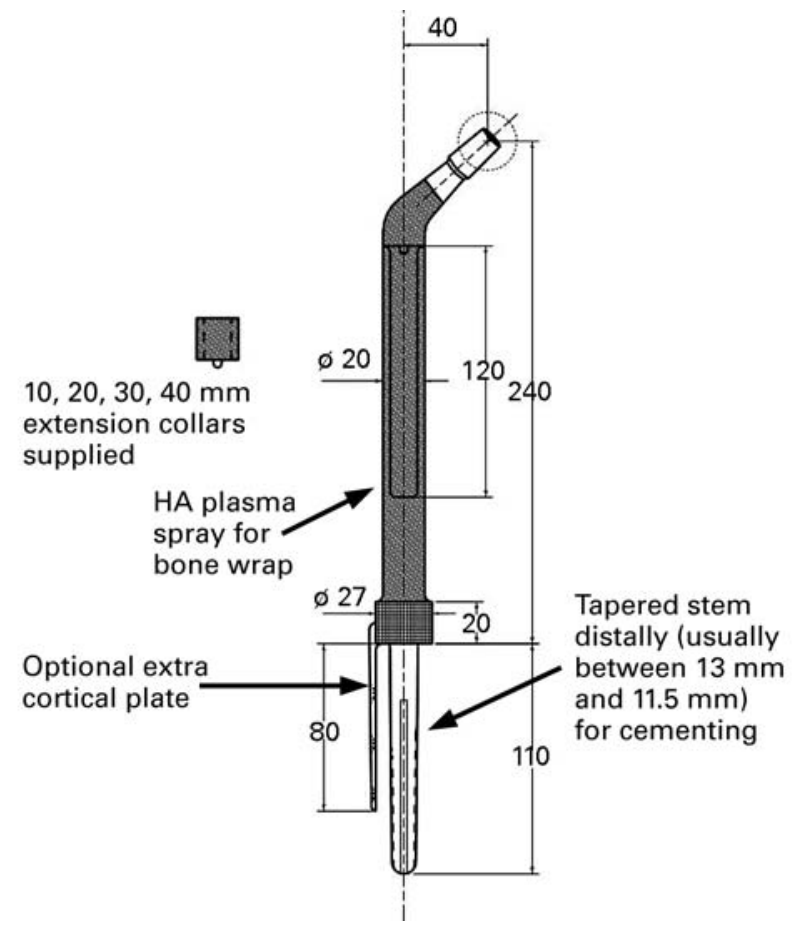

Fig. 1

Drawing showing an example of a typical internal proximal femoral replacement prosthesis (HA, hydroxyapatite). All values are given in $\mathrm{mm}$.

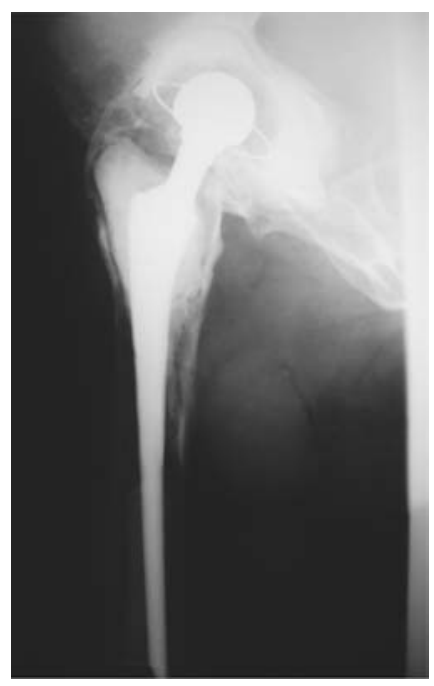

Fig. 2a

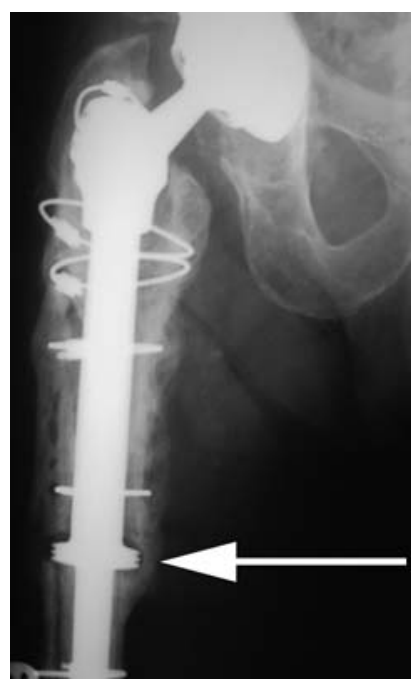

Fig. $2 b$
Anteroposterior radiographs of a 78-year-old woman who, prior to the index operation, was walking with two crutches and experiencing significant groin and thigh pain, a) failure after a third revision with gross osteolysis and little structural bone remaining pre-operatively, b) three years after surgery showing a well-fixed femoral prosthesis with excellent bone ingrowth on the HA collar (arrow) and the proximal part of the femoral prosthesis.

had been performed on the hip. Revision for aseptic loosening (Fig. 2) was carried out on 22 patients and in five the

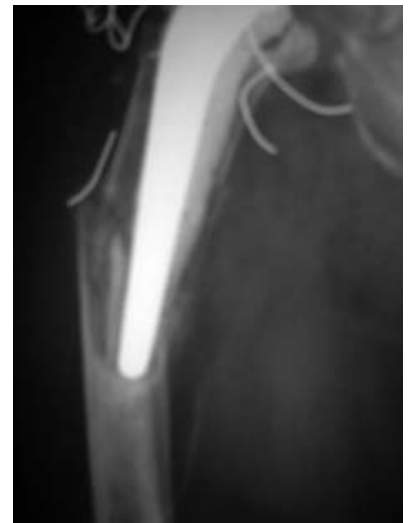

Fig. 3a

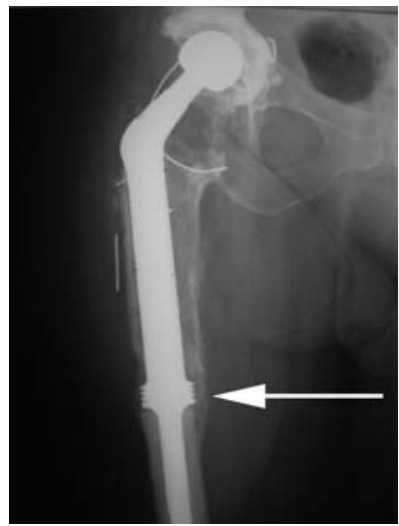

Fig. 3b
A 73-year-old man who was bedbound following failure of a first revision and peri-prosthetic fracture. a) Anteroposterior radiograph showing loss of bone stock and peri-prosthetic fracture, and b) one year after surgery, showing excellent fixation and bone induction around the HA collar of the custom-made prosthesis (arrow).

indication was a peri-prosthetic femoral fracture, usually with bone loss (Fig. 3). Three patients required distal-plate augmentation.

Of the 22 treated for aseptic loosening, three were Paprosky grade IIIB and 19 grade IV. In three of these, previous infection had been treated with resection arthroplasty and staged re-implantation once the infection had been eradicated.

Post-operatively, the patients were evaluated clinically and radiologically at six weeks, 12 weeks, six months, 12 months, and annually thereafter. At the last follow-up, the Harris and Oxford hip scores (HHS, OHS, respectively) were established by an independent reviewer (PKJ) who evaluated serial radiographs to grade the femoral defects, the stability of the implants and bone ingrowth. The same reviewer assessed osseointegration, and bone growth was evaluated by measuring the width in $\mathrm{mm}$ of the largest piece of bone in three zones of the proximal part of the implant and two zones around the collar on anteroposterior and lateral radiographs (Fig. 4). This was undertaken immediately after operation and on the last follow-up radiographs at a mean of 28 months (12 to 40). This enabled us to establish whether there was improvement in the proximal bone stock and ingrowth on the HA collar and where the improvement had occurred.

A telephone or postal questionnaire was filled in for those unavailable for clinical review. As defined by Gross et $a{ }^{1},{ }^{6}$ a successful outcome was an increase in the HHS of $\geq$ 20 points, a radiologically stable implant and no need for further femoral reconstruction.

Operative technique. With the patient in the lateral decubitus position, previous skin incisions were used or modified to allow an extended lateral transfemoral approach, including an extended trochanteric osteotomy. ${ }^{7,8}$ This was partic- 


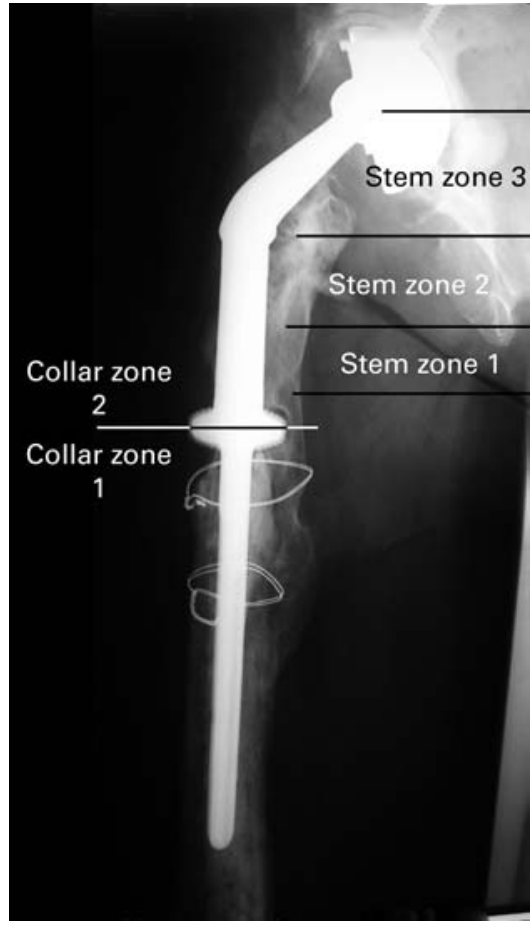

Fig. 4

Anteroposterior radiograph showing the measurements of bone growth and osseointegration. The width of the largest piece of bone adjacent to the zones was measured in the immediate post-operative anteroposterior and lateral radiographs and the last follow-up radiographs.

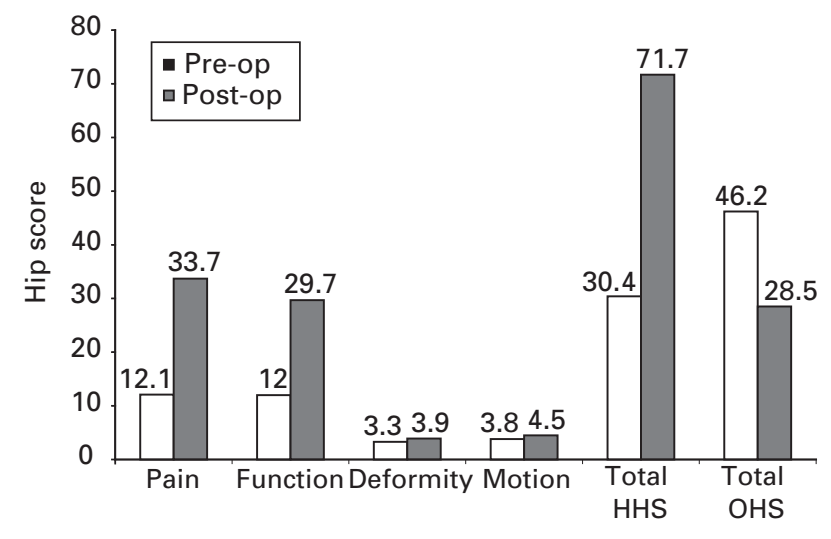

Fig. 5

Differences in pre- and post-operative Harris hip scores (HHS) and Oxford hip scores (OHS). The numbers above each bar indicate the functional score for each section.

ularly useful when removing a well-fixed stem or when the femoral component had fractured. It also facilitated rapid removal of retained cement. ${ }^{9}$ A transverse osteotomy was

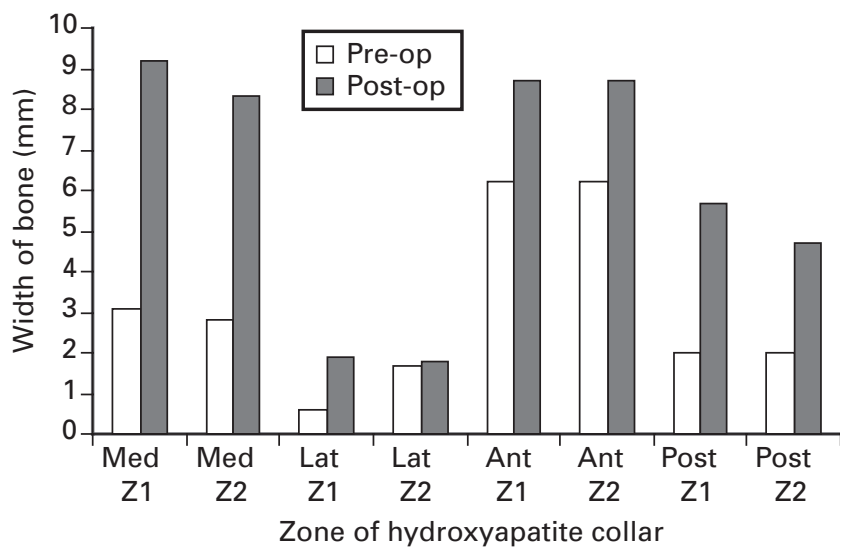

Fig. 6

Bone growth around the hydroxyapatite collar pre- and post-operatively (Z, zone; Med, medial; Lat, lateral; Ant, anterior; Post, posterior).

performed at the pre-determined site as described by Wagner. ${ }^{7,8}$ The distal femur was reamed progressively until the diaphysis was engaged using image intensifier guidance where necessary. A segment of bone was removed to allow fitting of the HA collar. If the femoral canal was narrow enough, a cement restrictor was used. This was not always possible if the canal was divergent. The prosthesis was cemented distally and the proximal shell of bone 'wrapped' around the HA-coated proximal stem and secured with Dall-Miles cables (Stryker Corporation, Kalamazoo, Michigan). Immediate distal fixation was achieved in all cases. In eight patients, the acetabular component was also revised.

Tissue samples were sent for Gram staining, culture and sensitivity studies. Peri-operative antibiotic prophylaxis consisted of routine intravenous cefuroxime followed by oral antibiotics until the wound was dry. Thromboprophylaxis was by elastic stockings, foot pumps and low molecular weight heparin (LMWH).

Post-operatively, the patients were mobilised as soon as possible. They were placed into 'slings and springs', and if abductor control was poor they were mobilised in an abduction brace. Walking, initially 'touch' weight-bearing, was commenced as soon as comfort allowed. After six weeks, the patients were assessed clinically and radiologically. If satisfactory, they were allowed to progressively increase weight-bearing.

For statistical analysis, SPSS (version 14.0; SPSS Inc., Chicago, Illinois) was used to perform $t$-tests to analyse differences in the HHS and OHS before and after the operation. A p-value of $<0.05$ was considered significant.

\section{Results}

At the time of review, four patients had died and one was lost to follow-up. Functional scores were therefore available for 22 patients at a mean follow-up of 55.3 months ( 25 to 126). In the four deceased patients the prosthesis 


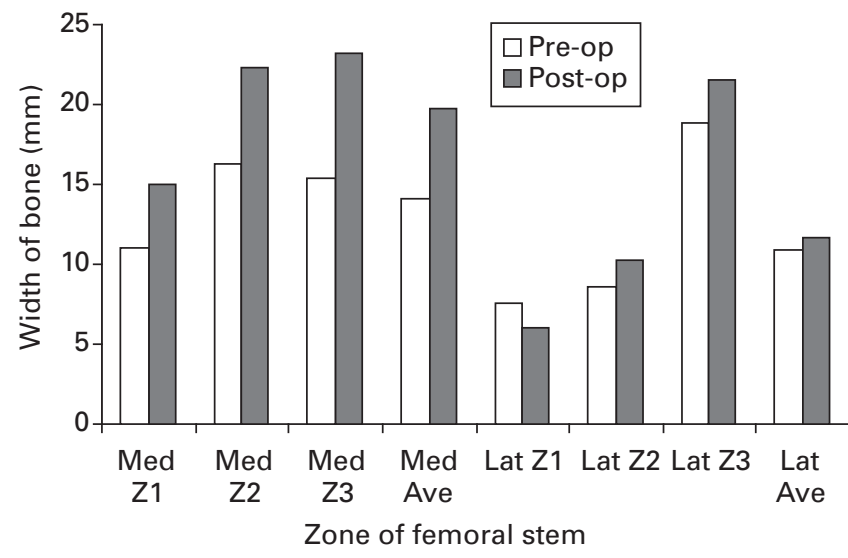

Fig. $7 \mathrm{a}$

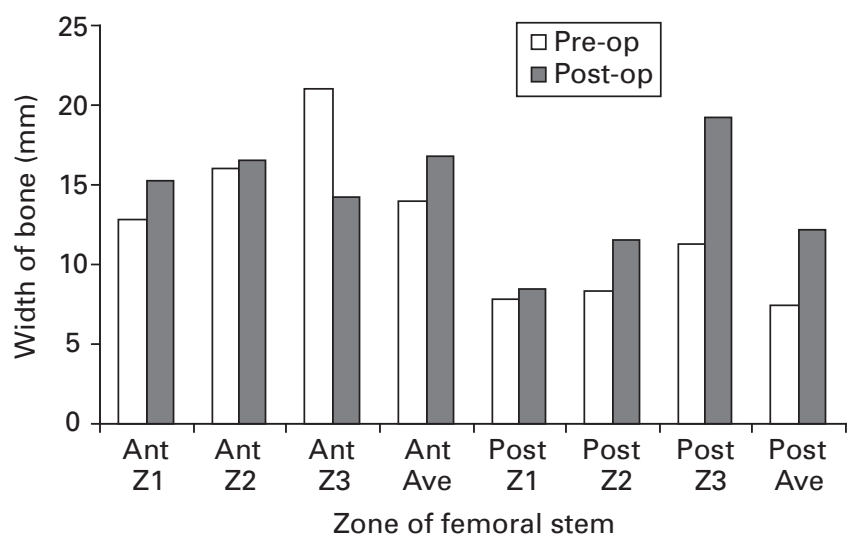

Fig. 7b

Graphs showing a) femoral stem bone growth on the anteroposterior radiograph, and b) femoral stem bone growth on the lateral radiograph preand post-operatively. Z, zone; Med, medial; Med Ave, average of the entire medial zone of the femoral stem; Lat, lateral; Lat Ave, average of the entire lateral zone of the stem; Ant, anterior; Ant Ave, average of the entire anterior zone of the stem; Post, posterior; Post Ave, average of the entire posterior zone of the stem.

remained well fixed. As determined by the criteria of Gross et al, ${ }^{6}$ the operation was successful in 19 of the 22 patients $(86.4 \%)$. Two had a decrease in HHS and one an increase of only nine points. The mean HHS increased from 30.4 (3 to 57.7) pre-operatively to 71.7 (44 to 99.7) postoperatively (paired t-test, $\mathrm{p}<0.001$; Fig. 5), a highly statistically significant mean increase of 41.3 points $(-8.2$ to 81 ) (95\% confidence interval (CI) 28.8 to 48.9 ). The two main improvements for the patients were in pain and function, though there were also modest improvements in deformity and motion (Fig. 5). The mean OHS decreased from 46.2 (38 to 60 ) to 28.5 (17 to 42 ) following the operation (paired t-test, $\mathrm{p}<0.001)$, with a mean decrease of 17.7 (-38 to +12$)(95 \%$ CI -22.3 to -13.3$)$.

There were no revisions of the femoral component for aseptic loosening. Figures 6 and 7 show that maximal bone growth occurs in the posteromedial aspect of the HA collar. There was a considerable increase in the width of bone around the medial aspect of the proximal stem (Fig. 7a) in all zones, but only proximally when assessing the posterior aspect of the stem (Fig. 7b). There was a small increase in width around the anterior and lateral aspects of the stem.

Dislocation occurred in two patients within three months of the operation and was managed initially by successful closed reduction under general anaesthesia. One required a change in the polyethylene liner and femoral head four months after the index procedure and functional scores were obtained three years after the revision operation. In the other patient, the acetabular component was revised two years after internal proximal femoral replacement and the HHS and OHS were evaluated 27 months after revision. Initially, both patients had simultaneous revisions of their acetabular components at the time of internal proximal femoral replacement.
There were three cases of deep infection, and at the time of the operation prophylactic antibiotics had been given in accordance with previous sensitivities. All three had previously suffered from infected prostheses and it was assumed the infection had been eradicated. Therefore, there may have been failure to eradicate the earlier infection rather than it be considered a complication of the revision surgery. Two patients were managed successfully by wound debridement and washout at one and three months, respectively, following internal proximal femoral replacement and are on long-term suppressive antibiotics. Their mean postoperative HHS was 62.8 and 68.9 at 39 and 119 months following surgery, respectively. One patient required a twostage revision procedure which has effectively eradicated the infection. The first stage was carried out 27 months after the initial proximal femoral replacement and the second eight weeks afterwards; 19 months later, his HHS was 47.5 .

\section{Discussion}

When there is severe loss of femoral bone stock in revision hip arthroplasty, the options are limited. Our only viable alternatives were a custom-made or modular proximal femoral endoprosthesis or a proximal femoral allograftprosthesis composite. ${ }^{4}$ Our early results are encouraging, and compared with the current literature, the complication rates are acceptable. ${ }^{10,11}$

There are no studies of large numbers of patients with grade IV defects. Sotereanos et $\mathrm{al}^{2}$ described 17 patients, only two had grade IV defects and the remainder were IIIB. These authors reported excellent results with a custommade, fully porous HA-coated femoral stem with holes for distal cross-locking. Our cohort of patients had more severe defects, but had similar increases in the HHS, albeit with higher complication rates. 
Wagner's concept of distal fixation, thereby allowing the proximal diseased part of the femur to heal, ${ }^{7,12}$ has been adopted by many surgeons undertaking revision hip arthroplasty. ${ }^{13,14}$ More recently, the Wagner cementless self-locking stem has been used for peri-prosthetic fractures. ${ }^{15}$ However, because of poor quality of the distal bone, this implant would not be suitable for our cohort of patients, particularly those with Paprosky grade IV defects, where distal fixation cannot be achieved without cement.

Saksena, Haddad and Muirhead-Allwood, ${ }^{16}$ using customised uncemented stems for revision total hip replacement, reported changes in functional scores similar to those in our study, but they did not discuss the Paprosky grade, and their use of uncemented stems suggests that their cohort had a lower grade of defect.

Modular stems, with their theoretical advantage of intraoperative versatility, are increasingly popular in revision hip arthroplasty. ${ }^{17}$ Chandler et al ${ }^{18}$ demonstrated significant increases in the HHS using the S-ROM (DePuy, Warsaw, Indiana) femoral stem, but their complication rates were high. They did not classify their femoral defects, but the prosthesis has been used in other studies where patients had less severe grade II/III defects. ${ }^{19}$

Allograft-prosthesis composites are generally reserved for reconstructing only the most severely-deficient proximal femora, and the initial results are good. ${ }^{20,21}$ However, this technique is technically demanding, expensive, and resorption and failure inevitably occur.

Using the criteria of Gross et al, ${ }^{6}$ our success rate was $86.4 \%$ (19 of 22 patients). Our prosthesis, augmented by the use of an HA collar, enhances extracortical bone integration within the implant surface. This makes use of the reactive bone formation that grows proximally from the transection site over the shaft of the implant to form a bony bridge. In our series, the majority of bone formation occurred around the medial, anterior and posterior aspects of the collar. Bony bridging is believed to reduce loosening by acting as a 'purse string' that seals the bone-implant interface, thereby preventing the migration of wear particles. $^{21-23}$ It is hoped that extracortical bridging in our patients may also lead to preservation of load transfer at the shoulder of the implant, thereby reducing stress within the cement and increasing implant survival.

There was an improvement in femoral bone stock around the HA-coated proximal stem. The highest increases in bone width occurred superomedially, which is the main load-bearing area. This study confirms that HA coating of the proximal part of the implant conveys the benefits of osseointegration and improves proximal bone stock. Saksena et $\mathrm{al}^{16}$ showed similar results using an HAcoated customised implant. The diversity of bone damage and loss in the proximal femur makes comparison of results difficult when evaluating studies of revision hip arthroplasty. In most series, when the results of extensively porous-coated stems have been compared with modular prostheses, there has been no difference. ${ }^{17}$
In our patients with Paprosky grade IIIB and IV defects and multiple previous operations, internal proximal femoral replacement enabled reconstruction of the most severe femoral defects, provided instant fixation to allow early mobilisation, and promoted biological integration and reconstitution. Preservation of the abductor mechanism potentially allows further improved function. Longer-term evaluation is awaited, but the initial results are encouraging.

No benefits in any form have been received or will be received from a commercial party related directly or indirectly to the subject of this article.

\section{References}

1. Della Vale CJ, Paprosky WG. Classification and an algorithmic approach to the reconstruction of femoral deficiency in revision total hip arthroplasty. J Bone Joint Surg [Am] 2003;85-A(Suppl 4):1-6.

2. Sotereanos N, Sewecke J, Raukar GJ, et al. Revision total hip arthroplasty with a custom cementless stem with distal cross-locking screws: early results in femora with large proximal segmental deficiencies. J Bone Joint Surg [Am]2006;88-A:1079-84.

3. Haddad FS, Masri BA, Garbuz DS, Duncan CP. Femoral bone loss in total hip arthroplasty: classification and preoperative planning. J Bone Joint Surg [Am] 1999;81-A:1483-98.

4. Della Vale CJ, Paprosky WG. The femur in revision total hip arthroplasty: evaluation and classification. Clin Orthop 2004;420:55-62.

5. Masri BA, Dominic Meek RM, Duncan CP. Periprosthetic fractures: evaluation and treatment. Clin Orthop 2004;420:80-95.

6. Gross $A E$, Hutchison CR, Alexeeff M, et al. Proximal allografts for reconstruction of bone stock in revision arthroplasty of the hip. Clin Orthop 1995;319:151-8.

7. Wagner H. Revision prosthesis for the hip joint in severe bone loss. Orthopade 1987;16:295-300 (in German).

8. Rinaldi E, Marenghi P, Vaienti E. The Wagner prosthesis for femoral reconstruction by transfemoral approach. Chir Organi Mov 1994;79:353-6.

9. Paprosky WG, Martin EL. Cemented stem failure requires extended trochanteric osteotomy. Orthopaedics 2003;26:28-9

10. Malkani AL, Sim FH, Chao YS. Custom made segmental femoral replacement prosthesis in revision total hip arthroplasty. Orthop Clin North Am 1993;24:727-33.

11. Haentjens $\mathbf{P}$, De Boeck H, Opdecam P. Proximal femoral replacement prosthesis for salvage of failed hip arthroplasty: complications in a 2-11 year follow-up study in 19 elderly patients. Acta Orthop Scand 1996;67:37-42.

12. Wagner H. A revision prosthesis for the hip joint. Orthopade 1989;18:438-53 (in German)

13. Stoffelen DV, Broos PI. The use of the Wagner revision prosthesis in complex (post) traumatic conditions of the hip. Acta Orthop Belg 1995;61:135-9.

14. Kolstad K, Adalberth G, Mallmin H, Milbrink J, Sahlstedt B. The Wagner revision stem for severe osteolysis: 31 hips followed for 1.5 to 5 years. Acta Orthop Scand 1996;67:541-4.

15. Lyu SR. Use of Wagner cementless self-locking stems for massive bone loss in hip arthroplasty. J Orthop Surg 2003;11:43-7.

16. Saksena J, Haddad FS, Muirhead-Allwood SK. Experience of using customized uncemented stems for revision total hip arthroplasty with severe femoral bone loss. J Bone Joint Surg [Br] 2003;85-B(Suppl 1):61.

17. Gurkan L, Wenz JF. Modular femoral implants for revision hip arthroplasty. Current Opinion Orth 2002;13:67-70.

18. Chandler HP, Ayres DK, Tan RC, Anderson LC, Varma AK. Revision total hip replacement using the S-ROM femoral component. Clin Orthop 1995;319:130-40.

19. Christie MJ, DeBoer DK, Tingstad EM, et al. Clinical experience with modular noncemented femoral component in revision total hip arthroplasty: 4 to 7-year results. J Arthroplasty 2000;15:840-8.

20. Haddad FS, Garbuz DS, Masri BA, Duncan CP. Structural proximal femoral allografts for failed total hip replacements: a minimum review of five years. J Bone Joint Surg [Br] 2000;82-B:830-6.

21. Blackley HRL, Davis AM, Hutchison CR, Gross AE. Proximal femoral allografts for reconstruction of bone stock in revision arthroplasty of the hip: a nine to fifteenyear follow-up. J Bone Joint Surg [Am] 2001;83-A:346-54

22. Ward W, Johnston K, Dorey F, et al. Extramedullary porous coating to prevent diaphyseal osteolysis and radiolucent lines around proximal tibial replacements: a preliminary report. J Bone Joint Surg [Am] 1993;75-A:976-87.

23. Kalia P, Blunn G, Miller J, et al. Do autologous mesenchymal stem cells augment bone growth and contact to massive bone tumor implants? Tissue Eng 2006;12:161726. 